Proc. Indian Acad. Sci. (Chem. Sci.), Vol. 105, No. 6, December 1993, pp. 359-371.

(C) Printed in India.

\title{
What's new in stereophotolithography?
}

\author{
J C ANDRE*, S CORBEL and J Y JEZEQUEL \\ GdR "Optical Instrumentation" CNRS, ENSIC-INPL, BP451-F 54001 Nancy Cedex, \\ France
}

\begin{abstract}
Laser stereophotolithography is a technology which allows the space-resolved phototransformation of a product. In this paper we describe the different basic processes with their limits. We present several ways to override these limits and discuss new application areas.
\end{abstract}

Keywords. Stereophotolithography; space-resolved phototransformation.

\section{Introduction}

First generation stereophotolithography (SPL) deals with the manufacture of threedimensional objects. They are made by space-resolved laser-induced polymerization. As a matter of fact, one uses a layer-by-layer process (photolithography) which allows the manufacture of objects (stereo). This scientific field, which is widely open to industrial application (design, prototyping, scale models etc.), is still restricted by the quality of the materials: volume shrinkage, warping, hardness, mechanical properties etc. All this defines a wide research area in connection with the making of new photoconvertible materials by the association of monomers, the addition of fillers and new photochemical initiators.

It is, for example, possible to override the limits of this technology, induced by volume variations, by modifying the manufacture process to take them into account beforehand. Also, since we aimed at manufacturing pieces fit for use, we developed a "second generation" technique that we describe in this paper.

In other respects, several different domains of application exist and every one of them needs specific materials as well as specific processes. They can be the manufacture of optical pieces, microtechnological objects, scale models etc. This proves that the market for stereophotolithography must expand and find application areas in specific domains. Out of this description, the use of new principles in photochemical initiation allows the possibility of 3D information systems.

In this paper, we shall recall the bases of the "first generation" stereophotolithography, show its limits and consider solutions to reach better results. These improvements will come from the materials and/or the processes.

\footnotetext{
* For correspondence
} 


\section{Recalls on the laser stereophotolithography}

We have developed, along with others, a process which makes three-dimensional objects by polymerizing a liquid monomer using a laser energy source (Andre et al 1990). The laser beam can be focused precisely on a liquid so that a liquid/solid photochemical transformation can be achieved. The advantages over the traditional techniques are that a mould is no longer necessary, the shape of the object can be totally arbitrary and wear of the tool is not needed.

Here, we show how three-dimensional objects can be created by using computeraided design. In the current stage, this new technique, which has led to very promising results, is limited by the choice of materials, and the traditional CAD has to be thus adapted. After discussing briefly the manufacture of objects by computer-aided laser polymerization, the coupling between the adapted CAD and the choice of materials is shown. The applications of this new technique are not all known yet; however, future progress will depend on the ability of specialists in the fields of materials engineering, photochemistry and computer-aided design to work together.

\subsection{Brief review of photopolymerization methods}

If a sufficient number of photons is absorbed per volume unit, unstable species are created and the liquid monomer polymerizes into a solid which is not soluble in the liquid monomer.

$$
\begin{gathered}
A+h v \rightarrow \text { unstable species, } \\
\text { unstable species }+M \rightarrow \text { nonsoluble polymers. }
\end{gathered}
$$

The above simplified mechanism shows that two main difficulties have to be surmounted: the local light absorption and the choice of materials. If these two main difficulties are only partially mastered, a modification of the computer-aided design becomes necessary.

2.1a Light absorption: Two excitation techniques have been proposed so far: multiphotonic absorption and monophotonic absorption. For now, the second seems more efficient for industrial applications.

The theoretical basis is the Beer-Lambert law. A light beam is incident on $z$, the concentration $C_{a}$ is such that the light absorption during $\mathrm{d} t$ produces a polymerization of the monomer between $z$ and $z+\Delta z$. If the beam radius is $\Delta r$, the polymerized voxel volume is $\pi \Delta r^{2} \Delta z$.

Successive layers of thickness, $\Delta z$, are made. This is shown qualitatively in figure 1 . Starting from the base which contains a layer of thickness $\Delta z$, the laser beam is displaced in the $x y$ plane in order to solidify the $z=0$ plane. Afterwards, a new layer of monomer is added and the process is repeated. An object can be made with just one laser. The laser beam is guided into the reactor containing the monomer. The guide must be very loosely attached to the created voxel otherwise deformation or rupture is possible. Several techniques enabling this exist. In all monophotonic processes, the object coordinates stored in the computer are given in the form $z=f(x, y)$.

2.1b Materials: Unless some special mechanical property of the object is desired, the liquid monomers should have the following characteristics: rapid reaction rate 


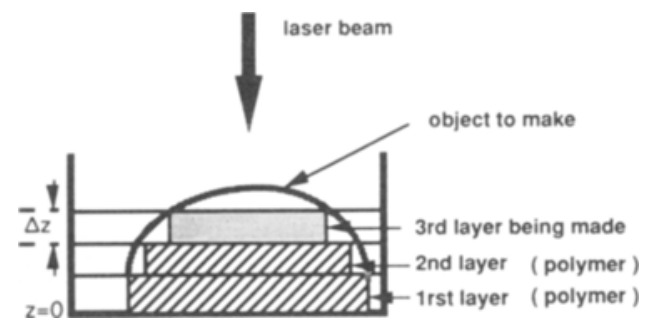

Figure 1. Principle of the layer-by-layer space-resolved polymerization.

and lowest possible viscosity in order to reduce the time needed per layer and to limit hydrodynamic side effects caused by the guide displacement. Monomers $\mathbf{M}$ having such properties are mainly in the epoxy or acrylate groups. Photochemists know well how to initiate and control the reactions using these species. When a monomer is polymerized, a volume variation is observed due to the photoreaction. This has various consequences. A polymerized voxel, which is not sustained and whose density is higher than that of the liquid, will tend to sink to the bottom unless held by surface tension forces. The manufactured object may thus acquire dimensions other than the ones specified. Furthermore, the object can deform.

In order to remedy these difficulties, materials which do not shrink and which have the properties mentioned earlier have to be searched for ( $c f \S 3)$. These materials exist but their viscosity is often too high. Another solution is to adapt the computeraided design in order to limit memory effects induced by the polymerization.

The principle consists in polymerizing voxels that are separated one from the other. The volume variation of each element occurs independently and then the interstices separating the voxels are polymerized. Since the laser has to be continuously displaced, a galvanometric mirror controlled by a computer should be used.

\subsection{Basis of a laser CAD machine}

2.2a The machine: The monophotonic process described earlier is split into several sub-systems which are:

- the computer system which includes the computer which receives the CAD data from another system. The computer system adaptes the data to define the optimum laser beam displacement;

- the photochemical reactor;

- the laser;

- a motor-driven optical mechanical unit with several degrees of freedom which shapes the light beam and then deflects it into the monomer to be polymerized;

- a motor-driven system which moves the object along the $z$ axis.

2.2b CAD software: In our case, the objects to be made are solids, either filled or hollow. Furthermore, great care must be taken in their manufacture since they are intended to be duplicated. A model is the more realistic that it accounts for the greater number of constraints of the object. 
The system configuration must be able to use CAD software which is commercially available. The interface must be conceived and should:

- verify that the object can be made;

- choose the best manufacturing order;

- decompose the object into voxels;

- predict and impede object deformation;

- command the whole process.

We have previously shown that it is not acceptable to create voxels that are independent of the partially created object.

As indicated before, the object can be manufactured at $z$ by laser-induced polymerization on the whole or part of the surface. The polymerization time is proportional to $h^{3}$ for an object of size $h$ and proportional to $h^{2}$ if only the surface is polymerized. It may be advantageous to polymerize the surface of the object only plus several elements inside it to increase its rigidity.

After taking the object out of the reactor, it contains liquid monomer. It can be used as such or a more complete polymerization can be obtained in an annex system. One must take into account that in this latter case, the object can deform due to shrinkage. The definition of the points where the object must be reinforced could be interesting. All these technical difficulties can be solved by adapting existing CAD software or by carefully choosing the materials used.

2.2c Optical mechanics: Carboxylic components are the most well-known and the most efficient photochemical reaction initiators. Their absorption spectra are centred

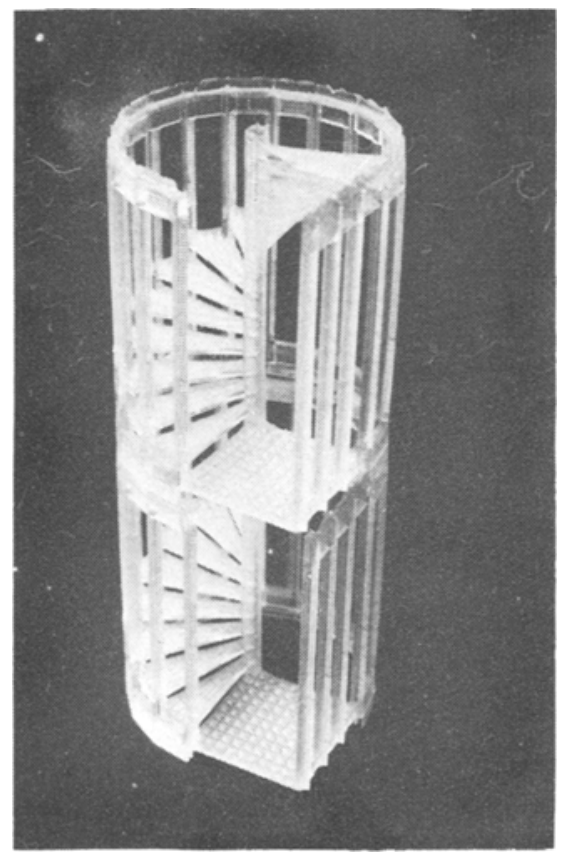

Figure 2. Object manufactured by laser stereophotolithography. 
at $350 \mathrm{~nm}$. A laser which emits in this spectral zone is usable $\left(\mathrm{He} / \mathrm{Cd}, \mathrm{Ar}^{+}\right.$etc.). Other compounds to initiate the reaction are available in the visible and the infrared regions.

When using $z$-axis displacement to guide the light beam, it is preferable to use stages moved by a computer-controlled step-by-step motor. For a system using the superposition of layers on the partially realized object, and in order to reduce manufacture time, it is advised to use displacement systems with very small inertia, for example, computer-controlled galvanometric mirrors.

This technique allows the manufacture of objects having a satisfying shape and quality ( $c f$ figure 2). Nevertheless, as already said, it is necessary to improve the process to make it completely satisfactory. The following paragraphs describe several ways to improve this process.

\section{Improvement of first generation processes}

It is clear that we must first improve the qualities of the materials. We arranged them in the following decreasing order of importance:

- volumic shrinkage;

- viscosity;

- mechanical and thermal properties;

- resins with fast reaction kinetics.

Since so far it has been impossible to obtain no volume variation between the monomer and the polymer when the monomer is viscous, we had to present the following modifications.

\subsection{Improvement of the resins used in stereophotolithography (SPL)}

It is necessary to use a resin whose shrinkage is as small as possible to avoid volume deformations and shear stresses in the objects manufactured by SPL.

Experiments prove that when we use oligomers instead of monomers, the shrinkage decreases. But since at the same time the viscosity of the liquid increases appreciably, this limits the interest of the process beyond some value (several Pa.s typically).

Table 1. Principal resins used in SPL commercially available in Europe.

\begin{tabular}{|c|c|c|c|c|c|c|}
\hline Resin & Density & $\begin{array}{c}\text { Viscosity } \\
(\text { Pa.s) } \\
\text { (at } \mathrm{T}^{\circ} \mathrm{C} \text { ) }\end{array}$ & Appearance & $\begin{array}{c}\text { Elongation } \\
\text { before break } \\
(\%)^{*}\end{array}$ & $\begin{array}{l}1 \% \text { secant } \\
\text { modulus } \\
(\mathrm{MPa})^{*}\end{array}$ & $\begin{array}{c}\text { Shrinkage } \\
(\%)\end{array}$ \\
\hline XB 5081 & $1 \cdot 14$ & $1.8-3.6(30)$ & Transparent & $2-3$ & $2500-3500$ & $5 \cdot 2$ \\
\hline XB 5134 & $1 \cdot 12$ & $1 \cdot 6-2(30)$ & $\begin{array}{l}\text { Slightly } \\
\text { opaque }\end{array}$ & 10 & $800-1100$ & \\
\hline $\begin{array}{r}\text { Somos } \\
3100\end{array}$ & $1 \cdot 13$ & $1.5(25)$ & $\begin{array}{c}\text { Amber } \\
\text { transparent }\end{array}$ & 15 & $800-900$ & $6 \cdot 2$ \\
\hline $\begin{array}{r}\text { Somos } \\
2100\end{array}$ & $1 \cdot 16$ & $5 \cdot 3(25)$ & $\begin{array}{c}\text { Milky white } \\
\text { opaque }\end{array}$ & 12 & $30-40$ & \\
\hline $\begin{array}{c}\text { Diacryl } \\
103\end{array}$ & $1 \cdot 12$ & $1 \cdot 0-1.4(25)$ & Transparent & $1 \cdot 2$ & & $6 \cdot 2$ \\
\hline
\end{tabular}

* Characteristics measured with products post-cured by fluorescent or UV light 


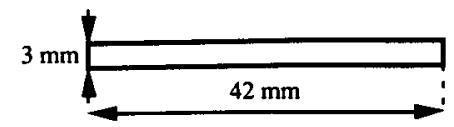

a
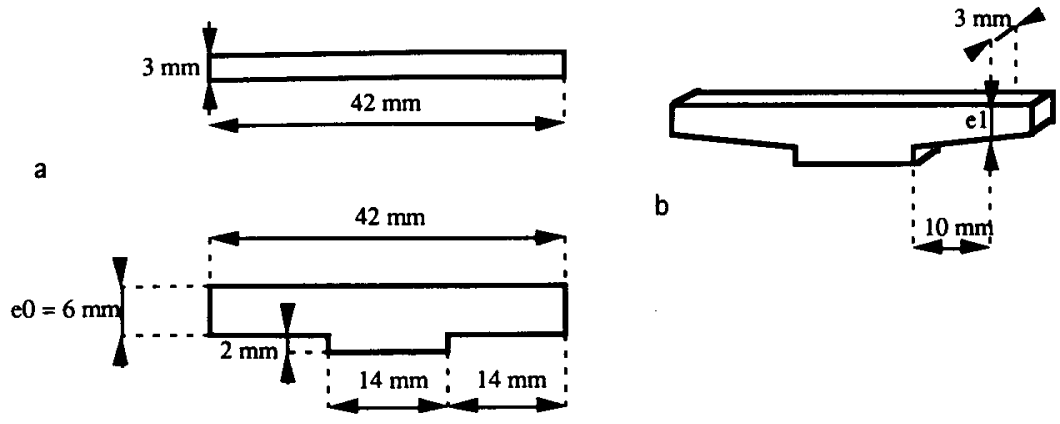

Figure 3. Test piece and bend coefficient. (a) Test piece. (b) Test piece manufactured by SPL.

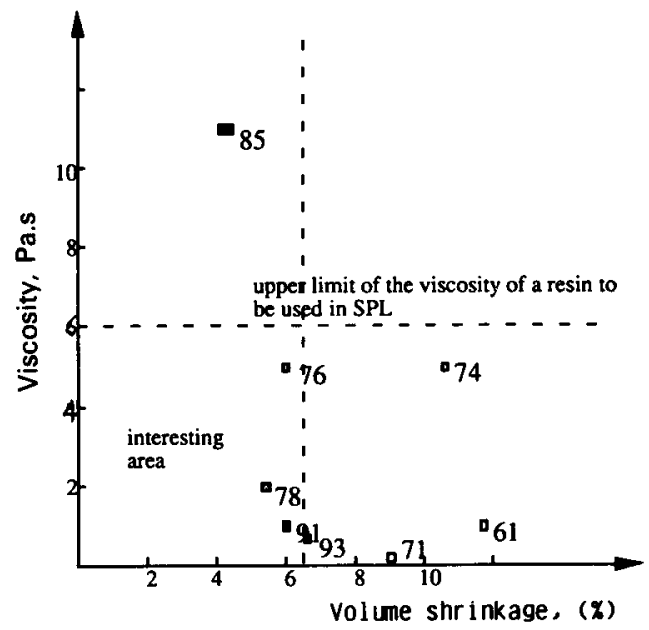

$\square c$ : resin having a bend coefficient of $c \%$. The higher this coefficient is, the darker the square is.

Figure.4. Influence of the viscosity and of the shrinkage on the bend coefficient $c$.

In table 1 are presented the characteristics of several resins commonly available commercially and used in Europe. They have small shrinkage ratios (between 5 and $6 \%$ usually).

Beside this shrinkage problem, it would be interesting to correlate the type of the resin with the global deformation of a test piece such as the one presented in figure 3 . These deformations occur after polymerization by SPL.

To compare various resins, we measure the thickness $e_{1}, 10 \mathrm{~mm}$ away from the base of the piece ( $c f$ figure 3 ). We define the bend coefficient $c$ (in \%) as the ratio:

$$
c=\left(e_{1} / e_{0}\right) \cdot 100
$$

where $e_{0}$ is the expected thickness of the piece. The closer $c$ is to 100 , the more precise is the piece.

By changing the composition of the reactive medium (a mixtare of several acrylic oligomers to have fast reaction kinetics), we have not managed as yet to reach 


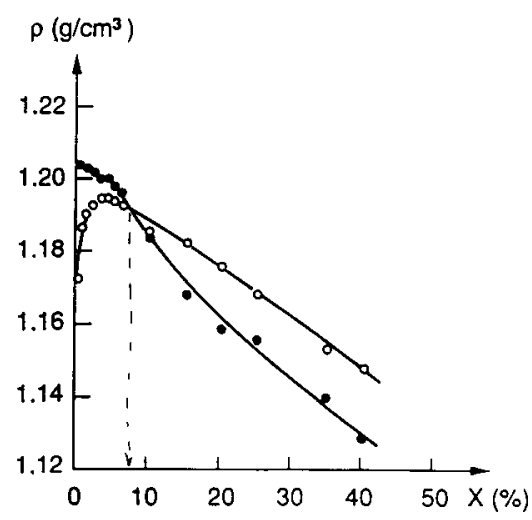

Figure 5. Specific gravity of the epoxy/PSS system before $(O)$ and after $(O)$ polymerization versus the PSS ratio.

shrinkage ratios smaller than $5 \%$ (cf figure 4). By a trial-and-error method, we made a resin (EOS-SPM) (Andre et al 1991), composed of aliphatic methane with carbonate acrylic which has not only these volume properties, but also reasonable mechanical characteristics.

Taking these results into account, a natural way to modify the characteristics of the reactive medium is to add charges in it. They limit the proportion of reactive medium per volume unit and then should induce smaller shrinkages. We experimentally found that the shrinkage decreases linearly with the charge ratio when the charge is not porous. This means that it can never be equal to zero. On the contrary, we experimentally proved that it was possible to obtain the same density for the monomer and the polymer ( $c f$ figure 5 ) when we used porous charges.

This method of research is very attractive as long as the resin viscosity does not exceed several pascals and as long as the sedimentation of the charge can be neglected. This implies the use of charges whose sizes are very small (Karrer et al 1992).

This short summary of the materials side of the process show that it is possible to improve the geometrical characteristics of an object manufactured by SPL by using proper compositions of the reactive medium. Nevertheless, it is still necessary to carry on investigations in this area, and this is why it remains one of our research fields of interest.

\subsection{Improvement of the process}

To improve the process, investigations can be developed in two directions: the improvement of the process itself and the improvement of the polymerization ratio to avoid a post-phototransformation.

Layerwise photopolymerization generally uses the principle of a polymerization by voxels which are independent from the others: in a given layer, the liquid-to-solid phototransformation is carried out on voxels which are individually affected by the shrinkage effect. Then, a second pass of actinic light allows the voxels to be sealed together so as to generate the final profile of the object. In doing so, the overall deformation is the result of reduced volume shrinkages corresponding to the association of the solid voxels with the others (cf figure 6). 


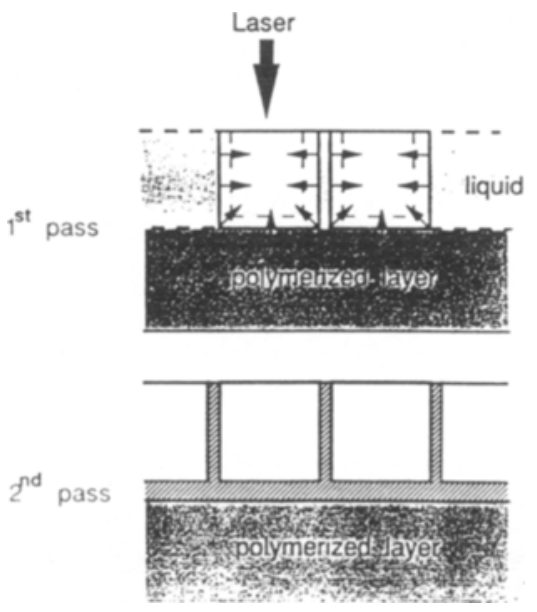

Figure 6. Principle of a space and time-resolved irradiation reducing the volume shrinkage.

A second improvement can come from research on materials having a high photopolymerization ratio, to avoid postpolymerizations in particular, which are very often the cause of global deformations. This postpolymerization is necessary when:

- the progress of the reaction is such that one is beyond the gel point. The mechanical properties of the object are satisfactory but complete polymerization is not yet achieved.

- only a part of the object being manufactured is photopolymerized in order to reduce the time of manufacture.

In table 2 we collected the conversion ratios of several commercialized acrylic resins that we measured.

Table 2. Experimental conversion ratio measurements for several commercialized resins.

\begin{tabular}{lcc}
\hline Resins & $\begin{array}{c}\text { Average conversion } \\
\text { ratios }(\%)\end{array}$ & $\begin{array}{c}\text { Energy density } \\
\left(\mathrm{mJ} . \mathrm{cm}^{-2}\right)\end{array}$ \\
\hline Mixture of & 98 & $81 \cdot 8$ \\
$50 \%$ ebecryl 7100 & & \\
$50 \%$ Cl993 & 76 & $81 \cdot 8$ \\
C1993 & 84 & $81 \cdot 8$ \\
Ebecryl 3200 & 90 & 20 \\
Mixture of & & \\
$50 \%$ ebecryl 3703 & & 20 \\
$50 \%$ Cl993 & 92 & \\
Mixture of & & $94 \cdot 6$ \\
$50 \%$ ebecryl 3703 & & 88 \\
$50 \%$ ebecryl 7100 & 46 & 16 \\
Ebecryl 140 & 91 & 16 \\
Eos SPM & 96 & \\
Ebecryl 7100 & 47 & \\
Diacryl 103 & & \\
\hline
\end{tabular}




\subsection{Conclusions}

We have qualitatively shown here the need to modify both the process and the phototransformable materials to improve the "first generation" stereophotolithography. Other principles can be considered to manufacture prototype pieces and the actually existing technology must still be improved to fill the market which could be its own.

\section{Second generation stereophotolithography}

The choice of naming this technology "second generation SPL" only deals with the fact that the object is self-sustained during its manufacture. It means that the nature and the reactivity of the materials are not taken into account in this definition. But in the following descriptions, it will on the contrary be natural to make a distinction between physical processes and chemical processes.

\subsection{Physical processes}

The basic principle of this process is the use of the powder of a material which can either melt or sinter. This material can be a polymer, a ceramic powder, metal powders etc. The choice of the material is much wider in that case because the imposed conditions are the following:

- the material must absorb light;

- the light energy must be high enough to melt or to sinter the powder;

- the adhesiveness between the particles of powder must be strong enough to obtain a solid object.

The principle of how such a system works is shown in figure 7 .

4.1a Principle of the manufacture of a layer: Let us assume that the piece has been manufactured upto height $h$. We make the mobile sustaining plate go down at a

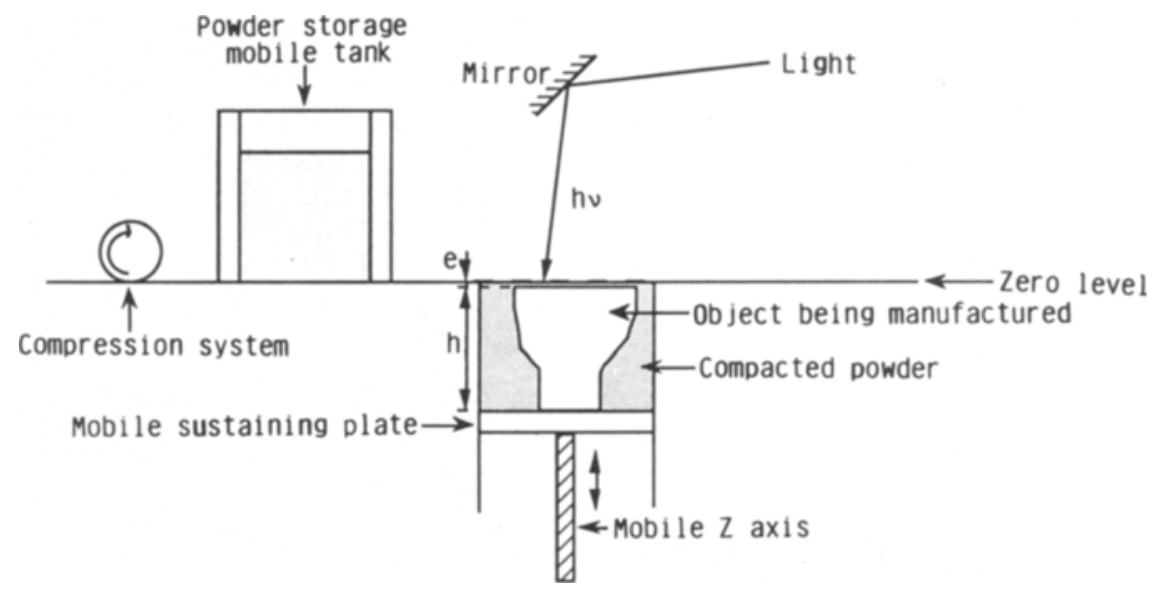

Figure 7. Schematic block diagram of a second generation SPL process using powder materials. 
distance $e^{\prime}$ from the zero level, slightly larger than the desired thickness $e$. In a second step, we slide the powder storage mobile tank over the sustaining plate. This translation of the storage tank fulfils two purposes: bringing in material and scraping the open surface of the reactor.

To bring the particles of powder closer together, we can move up the plate by a height of $\left(e^{\prime}-e\right)$ and compact the material to a maximum by the compression system. This more compact material leads to smaller shrinkages. The choice of the powder is very important for obtaining the smallest possible deformations. For example, the use of particles of different sizes could be a way to again increase the compactness.

In a third step - now that the new layer, $e$ thick, is ready - we can induce the phototransformation of the material by melting or sintering the powder. The expected space resolution depends on several parameters:

- the power of the laser;

- the resolution of the beam at the surface of the layer (one must in particular pay attention to the problems of reflection and of diffusion of the light which can be harmful to the eyes. The users of such apparatus must carefully read the safety notices about laser sources);

- the local distribution of the particles;

- the volumic and surface heat transfer coefficients;

- the thermal evolution of the material, from a pulverized state to a continuous state when melting occurs;

among others.

However, this technology in principle presents important advantages because the manufacture of an object made of different materials is highly simplified ( $c f$ figure 8).

When the manufacture of the part is achieved the part lies inside a compact matrix. The binding material is removed by a proper solvent - which can be water - and the powder that has not been irradiated is easily taken away.

We define the object inside the matrix as a 3D latent image and the removal of the non-irradiated powder as the development of this 3D image.

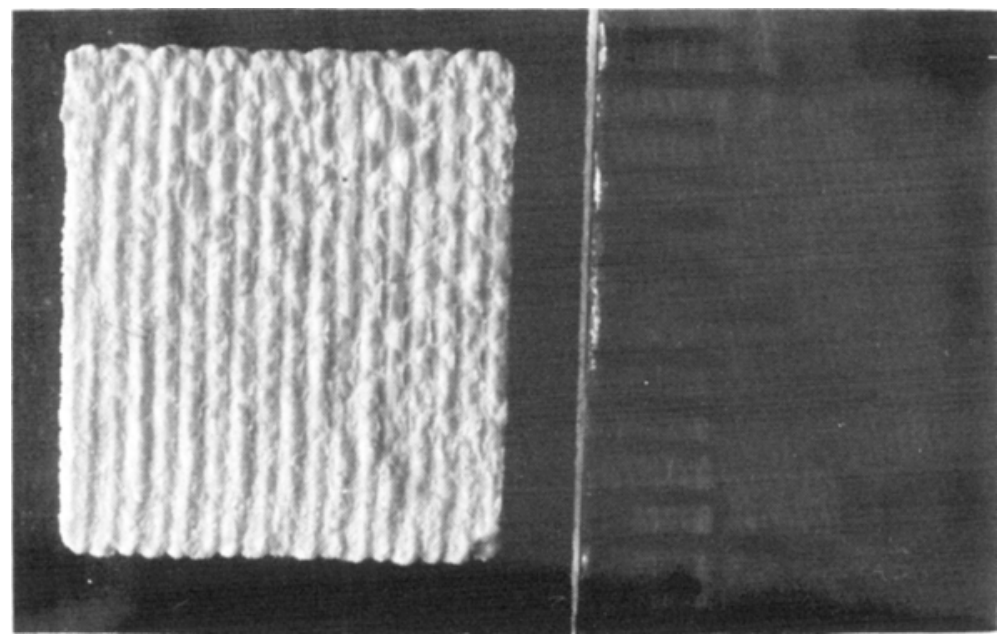

Figure 8. Piece manufactured by the space-resolved laser sintering of alumina powder. 


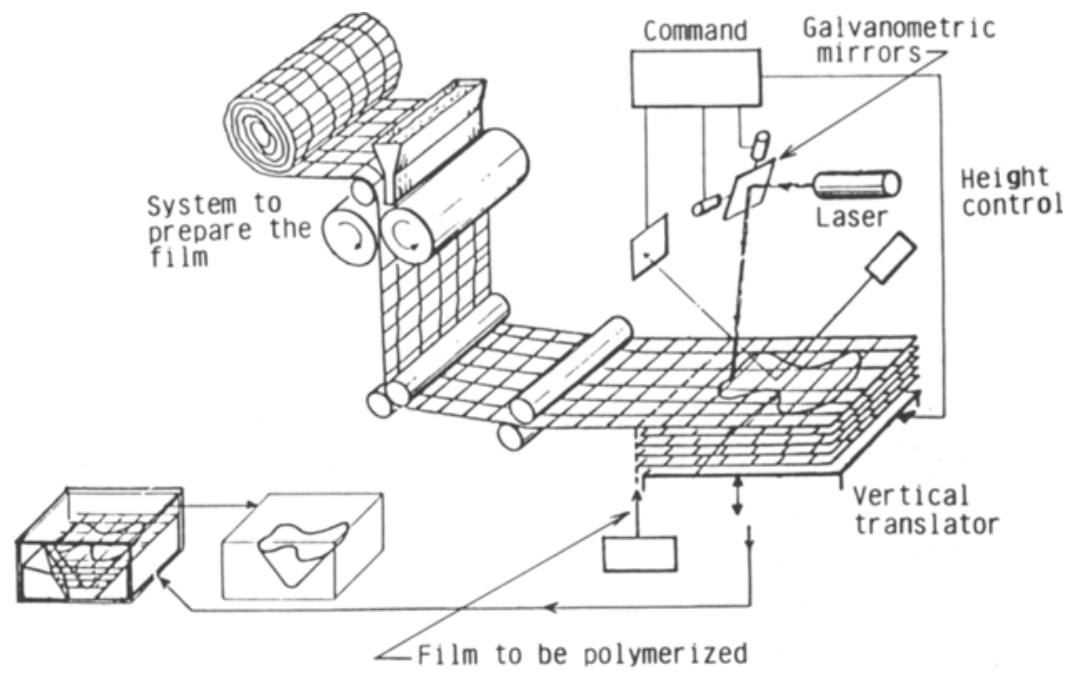

Figure 9. Second generation SPL apparatus using composite materials.

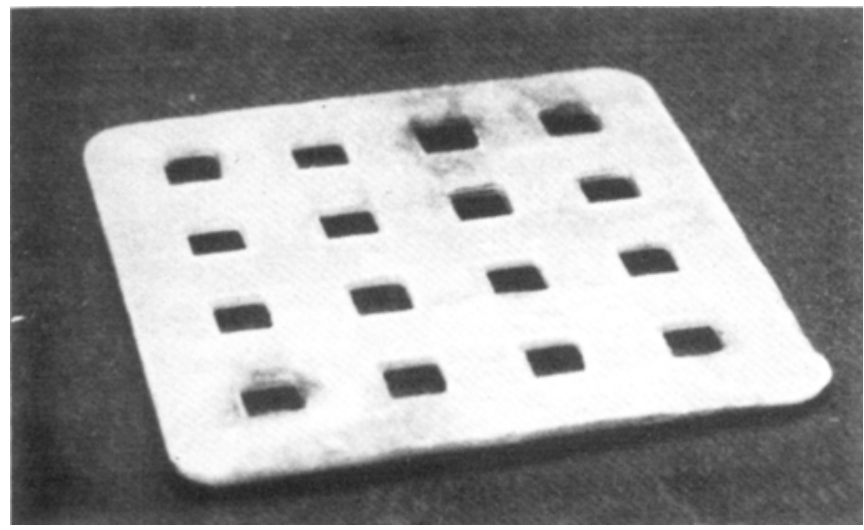

Figure 10. Object manufactured by a second generation SPL process.

\subsection{Chemical processes}

In the "bidimensional" lithography used in offset or in electronics, one uses photosensitive resins which are rendered insoluble by irradiation whereas the unradiated resin remains soluble in some solvent. This technique can schematically be compared to the photoreticulation of a part of the material, and we can apply the technique described in figure 7 to manufacture 3D objects: since the irradiated resin becomes insoluble, one only has to plunge the matrix into an appropriate solvent to develop the object.

Another method to manufacture objects by a second generation SPL process is to use a reinforced material made of wire netting embedded in a resin (with a possible added charge to try to minimise shrinkage). The principle of this process is depicted in figure 9 , and figure 10 is a photograph of an elementary object manufactured by this technique. 
When the object is achieved, the un-irradiated resin is removed by a solvent, as usual, and we get an object which is completely associated to the wire netting. The netting external to the object is destroyed by chemical treatment such as its dissolution by an acid or a base.

\section{Other processes - technological application fields}

This paper would become much too long if we described all the fields where a space resolved light absorption could be used to manufacture 3D objects. We shall nevertheless mention several attempts which have already been partly investigated.

\subsection{Information storage and third generation $S P L$}

The basic principle is the multiphonic absorption of light, either sequentially or simultaneously. Even if this process has not yet found any application in laser computer-aided design and manufacturing after the interesting research of the Battelle Institute, it should be possible to use it - as it is already the case with the photoablation processes (Andre 1991) to store information in 2D or even 3D.

\subsection{Optical-component manufacture}

One can focus a laser beam on a monomer to photopolymerize it and to manufacture optical components that way: for example microlenses, lenses or optical fibres (Brulle 1992). As an example, figure 11 depicts a process to manufacture optical fibres by space-resolved photopolymerization.

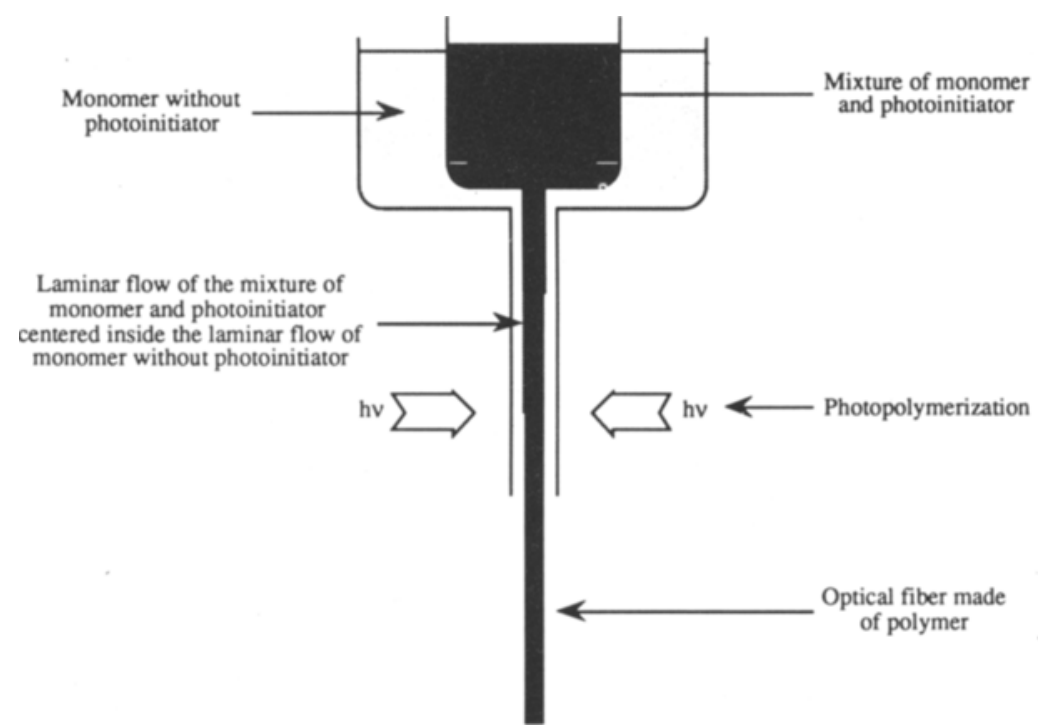

Figure 11. Manufacture of polymer optical fibres by the photopolymerization of a laminar flow of monomer. 


\subsection{SPL and microtechniques}

A new market which needs the manufacture of micro-objects is coming up: micromotors, active microsystems, microresonators etc. Studies on photonic systems able to manufacture active microobjects by SPL is of great interest because it opens up the possibility of making them automatically, via collective processes. These studies are far from completion but several technologies, based on the ones we described in this paper or on others (Meermann 1992), have already provided very encouraging results.

\section{$5.42 D$ photography}

The idea of combining the transformation of a material with its colouring, at least on the surface of the object, should allow the manufacture of 3D photographs. Since these studies are still under progress, we cannot describe them as yet.

\section{Conclusions}

Laser stereophotolithography is a recent technology which has been commercialized. It has not yet realised its entire potential, even if we do visualize some of them. If it is true that this technology will not probably take the place of all computer-aided tooling techniques, it is also true that all its applications are not yet known. To know them, it is necessary to undertake pluridisciplinary studies getting together photochemists, specialists in material sciences, automatics, informatics and possible users. This is a difficult but very stimulating activity, and we expect to persist in doing it.

\section{References}

Andre J C 1991 Microstéréophotolithographie. In Recherches en Microtechniques; réalités et perspectives. Institut des Microtechniques Ed., Besancon, France, pp. 73-88

Andre J C, Balmes F, Corbel S, Vanoeuvre F and Langer H 1991 Lichthärt bares Kunstoffmaterial, Deutschespatent 4138309

Andre J C, Corbel S, Nonnenmacher F, Schaeffer P and Cabrera M 1990 Computer aided manufacture of three-dimensional objects in Industrial Photochemistry - B, 475-95, ENSIC Ed. Nancy - France

Brulle Y 1992 Photonical processes for optics. Ph D thesis, Nancy, France

Karrer P, Corbel S, Andre J C and Lougnot D J 1992 Shrinkage effects in photopolymerizable resins containing filling agents: Application to stereophotolithography. J. Polym. Sci. 3042715

Meermann H 1992 Eiffeltower mit dem Laser gebaut. In MPG Presse Information, München-BDR, PRI C $3 / 92(12)$ 